\title{
Paciente em tratamento de neoplasia mamária sem hiperexperssão her-2, evoluindo com cardiotoxicidade, disfunção ventricular esquerda sintomática
}

\author{
Patient in treatment of mammary neoplasia without her-2 hyperexperssion, evolving with \\ cardiotoxicity, symptom left ventricular dysfunction
}

Paciente en tratamiento de neoplasia mamária sin hiperexperssión her-2, evoluyendo con cardiotoxicidad, disfunción ventricular izquierda sintomática

Paulo Ricardo Nauar Lisboa de Oliveira1, Vitor Bruno Teixeira de Holanda', Lucianna Serfaty de Holanda $^{1 *}$, Carla Daniele Nascimento Pontes ${ }^{2}$, Yuri José Almeida da Silva ${ }^{2}$, Ivy Almeida Cavalcante e Silva ${ }^{2}$.

\section{RESUMO}

Objetivo: Descrever o caso de uma paciente portadora de neoplasia de mama, atendida em Hospital privado, referência em oncologia, na cidade de Belém-PA, que evoluiu com cardiotoxicidade, insuficiência cardíaca sintomática, classe funcional IV da NYHA, durante tratamento específico. Método: Trabalho transversal, descritivo, do tipo Relato de Caso, obtido através de prontuário. Detalhamento do caso: Mulher, 45 anos, parda, natural de Belém-Pa, sem cardiopatia prévia. Diagnosticada com carcinoma mamário invasivo, submetida há 1 ano à quadrantectomia direita, com quimioterapia (QT) neodjuvante (esquema AC por 4 ciclos - Adriamicina e Ciclofosfamida e posteriormente Paclitaxel por 4 ciclos. Imunohistoquímica: HER-2 escore 1, receptor hormonal positivo e KI 67 10\%. Durante acompanhamento, realizou-se dosagem de biomarcadores (troponina) - negativos. A paciente teve boa evolução clínica, chegando a classe funcional I e reversão da disfunção ventricular esquerda (FEVE 56\%), após 6 meses de tratamento específico, que foi mantido por tempo indeterminado, por tratar-se de cardiotoxicidade tipo I. Conclusão: O desenvolvimento de protocolos, específicos, nas diversas instituições de nossa região, sejam públicas ou privadas, são fundamentais para o manejo adequado dos doentes. A interação entre a cardiologia e a oncologia contribui para a melhor evolução dos pacientes, tendo como objetivos principais a adoção de estratégias de prevenção.

Palavras-chave: Insuficiência Cardíaca, Oncologia, Cardiologia.

\section{SUMMARY}

Objective: To describe the case of a patient with breast neoplasia, attended at a private hospital, a reference in oncology, in the city of Belém-Pa, who developed cardiotoxicity, symptomatic heart failure and NYHA functional class IV during specific treatment. Method: Cross-sectional, descriptive work, of the Case Report type, obtained through medical records. Case detail: Female, 45 years old, brown, native of Belém- $\mathrm{Pa}$, without previous heart disease. Diagnosed with invasive mammary carcinoma, submitted to neuromuscular chemotherapy (QT) for 4 cycles - Adriamycin and Cyclophosphamide and subsequently Paclitaxel for 4 cycles. HER-2 score 1, hormone receptor positive and KI The patient had a good clinical evolution, reaching functional class I and reversion of left ventricular dysfunction (LVEF 56\%), after 6 months of specific treatment, which was maintained for an indefinite period, due to the type I cardiotoxicity. Conclusion: The development of specific protocols in the various institutions of our region, whether public or private, are fundamental for the proper management of patients. cardiology and oncology contributes to the better evolution of patients, with the main objectives being the adoption of strategies of prevent.

Keywords: Cardiac insufficiency, Oncology, Cardiology.

\footnotetext{
${ }^{1}$ Hospital Saúde da Mulher (HSM), Belém-PA. *E-mail: lu serfaty@hotmail.com

${ }^{2}$ Fundação Santa Casa de Misericórdia do Pará (FSCM-PA).
} 


\section{RESUMEN}

Objetivo: Describir el caso de una paciente portadora de neoplasia de mama, atendida en Hospital privado, referencia en oncología, en la ciudad de Belém-Pa, que evolucionó con cardiotoxicidad, insuficiencia cardiaca sintomática, clase funcional IV de la NYHA, durante tratamiento específico. Método: Trabajo transversal, descriptivo, del tipo Relato de Caso, obtenido a través de prontuario. Detalle del caso: Mujer, 45 años, parda, natural de Belém-Pa, sin cardiopatía previa. En la mayoría de los casos, la mayoría de las personas que sufren de cáncer de próstata, se han visto afectadas por el uso de la vacuna contra el virus de la inmunodeficiencia humana (VIH). El paciente tuvo una buena evolución clínica, llegando a la clase funcional I y la reversión de la disfunción ventricular izquierda (FEVE 56\%), después de 6 meses de tratamiento específico, se observó una dosificación de biomarcadores (troponina) que se mantuvo por tiempo indefinido, por tratarse de cardiotoxicidad tipo I. Conclusión: El desarrollo de protocolos, específicos, en las diversas instituciones de nuestra región, sean públicas o privadas, son fundamentales para el manejo adecuado de los enfermos. cardiología y la oncología contribuye a la mejor evolución de los pacientes, teniendo como objetivos principales la adopción de estrategias de pr evenção.

Palabras Clave: Insuficiencia Cardiaca, Oncología, Cardiología.

\section{INTRODUÇÃO}

O câncer de mama é o câncer mais frequente em mulheres no Brasil e no mundo, chegando a atingir cerca de $25 \%$ da população mundial (FRELAY J, et al., 2013).

Quimioterapia para pacientes com câncer de mama é um esquema potencialmente cardiotóxico, sendo de grande importância a identificação precoce e intervenção adequada em pacientes de risco. (FILHO R, et al., 2011).

Desde o decimo segundo Gallen International Breast Cancer Conference Expert Panel, em 2011 foram orientadas pesquisas com Imnunohistoquimica para a mama HER-2 (receptor de fator de crescimento epidérmico humano, ERBB2) e outros receptores que pudessem gerar novos tratamentos com anticorpos monoclonais e novos quimioterápicos (SILVA, 2016).

Quanto a cardiotoxicidade das antraciclinas (doxorrubicina, epirrubicina e idarrubicina), sabe-se que o dano é maior com dose maior cumulativa. Administrações contíbuas de antraciclinas geram lesão em miócitos cardíacos e no interstício, principalmente quanto maior for a dose, o que pode também estar associado a disfunção diastólica precoce e disfunção sistólica tardia (YET ET, et al., 2004).

Disfunção diastólica por toxicidade cumulativa dose dependente pode ser observada com dose cumulativa equivalente a $200 \mathrm{mg} / \mathrm{m} 2$, enquanto disfunção sistólica é observada usualmente com doses acima de 400 $\mathrm{mg} / \mathrm{m} 2$, com variabilidade segundo limiar individual. Entretanto, prejuízo na função diastólica foi observado com dose cumulativa de apenas $120 \mathrm{mg} / \mathrm{m}$. Complicações maiores ocorrem, especialmente, quando associadas a inibidores HER-2 -trastuzumabe (FRELAY J, et al., 2013).

Portanto, este estudo tem como objetivo descrever o caso de uma paciente portadora de neoplasia de mama, atendida em Hospital privado, referência em oncologia, na cidade de Belém-PA, que evoluiu com cardiotoxicidade, insuficiência cardíaca sintomática, classe funcional IV da NYHA, durante tratamento específico.

\section{RELATO DE CASO}

J.I.L.M 45 anos, parda, natural de Belém-PA, sem cardiopatia prévia. Diagnosticada com carcinoma mamário invasivo, submetida há 1 ano à quadrantectomia direita, com quimioterapia (QT) neodjuvante (esquema AC por 4 ciclos - Adriamicina dose total $240 \mathrm{mg} / \mathrm{m}^{2}$ e Ciclofosfamida totalizando $2400 \mathrm{mg} / \mathrm{m}^{2}$ e posteriormente Paclitaxel por 4 ciclos, totalizando $700 \mathrm{mg} / \mathrm{m}^{2}$, com término em janeiro de 2015), Imunohistoquímica: HER-2 escore 1, receptor hormonal positivo e KI 67 10\%. 
Em fevereiro de 2016 realizado mais um ciclo de QT com Paclitaxel $80 \mathrm{mg} / \mathrm{m}^{2}$, que não deu continuidade. Após ciclo complementar do taxano, desenvolveu sintomas de congestão pulmonar e edema, evoluindo em classe funcional IV da NYHA. Eletrocardiograma com taquicardia sinusal e padrão de Bloqueio de Ramo Esquerdo (BRE) novo.

Ecocardiograma transtorácico revelou disfunção ventricular esquerda (DVE) de grau importante (FEVE 26\%). Interrompida a quimioterapia e instituído tratamento padrão com enalapril e furosemida, espironolactona e digital. Posteriormente, introduzidos carvedilol e ivabradina.

Quadro 1 - Ecocardiograma da paciente demonstrando comprometimento cardíaco, Belém - Pará, 2018.

\begin{tabular}{|l|l|}
\hline Ecocardiograma Inicial 02/03/2016 & Ecocardiograma final 31/08/2016 \\
\hline AE $45 \mathrm{~mm}$ & AE $36 \mathrm{~mm}$ \\
VE $57 / 50 \mathrm{~mm}$ & VE $57 / 39 \mathrm{~mm}$ \\
FEVE (Simpson) 26\% & FEVE (Simpson) $56 \%$ \\
Disf. Diastólica: pseudonormal. & Disf. Diastólica Grau I \\
Mitral: refluxo moderado & Mitral: refluxo discreto \\
Tricúspide: refluxo importante & Tricúspide: refluxo discreto \\
PAP $50 \mathrm{mmHg}$ & Sem sinais sugestivos de HAP \\
\hline
\end{tabular}

Fonte: Dados da Pesquisa, 2018.

Retornos semanais no ambulatório de cardioncologia para ajustes terapêuticos e estabilização clínica. Durante acompanhamento, realizou-se dosagem de biomarcadores (troponina) - negativos. A paciente teve boa evolução clínica, chegando a classe funcional I e reversão da disfunção ventricular esquerda (FEVE 56\%), após 6 meses de tratamento específico, que foi mantido por tempo indeterminado, por tratar-se de cardiotoxicidade tipo I.

\section{DISCUSSÃO}

As complicações cardiovasculares são frequentes nos pacientes oncológicos, em especial nos casos de câncer de mama (EWER et al., 2005). Ressalte-se que, certos efeitos deletérios do tratamento quimioterápico no músculo cardíaco, podem determinar impacto significativo na sobrevida, resultando em aumento de morbidade e mortalidade (STEWART et al., 2001).

Dentre as diversas manifestações clínicas da cardiotoxicidade, a mais temida é a disfunção ventricular esquerda, levando a insuficiência cardíaca congestiva (SENGUPTA et al., 2008)

O presente relato, descreveu o caso de uma paciente, utilizando doses padrões de quimioterápicos, que evolui com cardiotoxicidade severa e insuficiência cardíaca sintomática, em inconformidade, aos dados descritos na literatura. Daniel Von Hoff, descreveu inicialmente a cardiotoxicidade em pacientes expostos a doses altas de doxorrubicina (> $450 \mathrm{mg} / \mathrm{m}^{2}$ ), ainda na década de 70 (SILVA 2016).

O aparecimento de disfunção ventricular, não é comum em pacientes com baixas doses de antracíclicos, porém quando ocorre, deve ser diagnosticada e tratada da forma mais precoce possível, uma vez que 0 aparecimento dessa complicação pode determinar interrupção do tratamento quimioterápico e dificultar a cura ou o adequado controle do câncer. É válido ressaltar que a insuficiência cardíaca tem pior prognóstico que muitas neoplasias e pode comprometer seriamente a evolução do paciente em tratamento (KY et al., 2014). 


\section{CONCLUSÃO}

O desenvolvimento de protocolos específicos nas diversas instituições do país, sejam públicas ou privadas, é fundamental para o manejo adequado dos doentes. A interação entre a cardiologia e a oncologia contribui para a melhor evolução dos pacientes, tendo como objetivos principais a adoção de estratégias de prevenção, o diagnóstico precoce e o tratamento das doenças cardiovasculares nessa população.

\section{REFERÊNCIAS}

1. FERLAY J, SOERJOMATARAM I, ERVIK M et al. Cancer Incidence and Mortality Worldwide: IARC CancerBase No. 11 Lyon, France: International Agency for Research on Cancer; GLOBOCAN 2012 v1.0, 2013.

2. KY B, PUTT M, SAWAYA H et al. Early Increases in Multiple Biomarkers Predict Subsequent Cardiotoxicity in Patients With Breast Cancer Treated With Doxorrubicin, Taxanes and Trastuzumab. J Am Coll Cardiol. 2014 March 4;63(8):809-816.

3. YEH ET, TONG AT, LENIHAN DJ et al. Cardiovascular complications of cancer therapy: diagnosis, pathogenesis, and management. Circulation. 2004;109(25):3122-31.

4. SENGUPTA PP, NORTHFELT DW, GENTILE F et al. Trastuzumab-induced cardiotoxicity: heart failure at the crossroads. Mayo Clin Proc. 2008;83(2):197-203.

5. CHEN T, XU T, LI Y, et al. Risk of cardiac dysfunction with trastuzumab in breast cancer patients: a meta-analysis. Cancer Treat Rev.2010 Oct 15. [Epub ahead of print].

6. EWER MS, LIPPMAN SM. Type II chemotherapy-related cardiac dysfunction: time to recognize a new entity. J Clin Oncol. 2005;23(13):2900-2.

7. STEWART S, MACINTYRE K, HOLE DJ, et al. More malignant than cancer? Five-year survival following a first admission for heart failure. Eur J Heart Fail. 2001;3(3):315-22.

8. SENGUPTA PP, NORTHFELT DW, GENTILE F, et al. Trastuzumab-induced cardiotoxicity: heart failure at the crossroads. Mayo Clin Proc. 2008;83(2):197-203.

9. EWER MS, EWER SM. Cardiotoxicity of anticancer treatments: what the cardiologist needs to know. Nat Rev Cardiol. 2010;7(10):564-75.

10. KALIL FILHO R, HAJJAR L, BACAL F, et al. I Diretriz Brasileira de Cardio-Oncologia da Sociedade Brasileira de Cardiologia. Arq Bras Cardiol 2011; 96 (2 supl.1): 1-52.

11. SILVA ERA. Cardiotoxicidade associada ao tratamento adjuvante com trastuzumabe em pacientes com carcinoma de mama. UNIVERSIDADE ESTADUAL DE CAMPINAS - FACULDADE DE CIÊNCIAS MÉDICAS CAMPINAS - SP - 2016. 